\title{
Screening citrus SSR molecular marker primers
}

\author{
Xiaojia Wang ${ }^{1, a}$, Zhihui Wang ${ }^{1, b}$, Siyu Zhang ${ }^{1, c}$, Jundou Liu ${ }^{2, d}$, Xun Wang ${ }^{3, e}$ \\ ${ }^{1}$ College of Horticulture, Sichuan Agricultural University, Chengdu, Sichuan, China \\ 2 The Agricultural Institute of Sichuan Province, Chengdu, Sichuan, China \\ ${ }^{3}$ Institute of Pomology \& Olericulture, Sichuan Agricultural University, Chengdu, Sichuan, China \\ amajiayan3303@163.com,b961124698@qq.com,c1285850704@qq.com \\ d112885608@qq.com, ${ }^{\mathrm{d}}$ wangxun0104@hotmail.com
}

Xiaojia Wang and Zhihui Wang contributed equally to this work.

Keywords: Citrus;SSR-PCR system;Primer screening;Cluster analysis.

Abstract. Within 38 primer pairs tested, 26 pairs were successfully amplified, and 81 PCR bands were amplified, with an average value of 3.12 bands per primer, 18 pairs showed $100 \%$ polymorphic ratio, 2 pairs showed $66.67 \%$ polymorphic ratio. In total, 72 PCR polymorphic bands were determined, and in average, 2.77 polymorphic bands were amplified by one primer pair. Twenty primer pairs were screened out, as their PCR bands were distinct, stable and polymorphic. Six citrus materials were clustered via genetic similarity coefficient using 20 pairs of primers screened out, 'Eureka lemon' and 'Ichang papeda' were clustered into one group, and then meet with 'Ponkan' and 'Nanfeng tangerine'. Meanwhile, 'Gaoban pummelo' and 'Kiyomi' were clustered into another group.

\section{Introduction}

In the long-term cultivation and cultivation, many unique local citrus germplasm resources have been formed due to various geographical, climatic and natural conditions. Most of these germplasm resources are formed by natural hybridization and natural buds during the evolution process. The genetic background is complex and difficult to define in classification. In the breeding of modern citrus varieties, it is one of the effective ways to use the excellent traits of local germplasm resources or to improve local germplasm resources ${ }^{[1]}$. SSR (Simple Sequence Repeats) is one of the most effective molecular marker techniques at present. It is widely used in the research of gene location, QTL, DNA fingerprint construction and genetic diversity analysis ${ }^{[2-5]}$.

This experiment is aimed at the recent publication of several SSR primers, which are amplified, analyzed and screened by six well-defined citrus materials to construct a highly efficient SSR molecular marker primer library for the upcoming local endemic citrus.

\section{Materials and Methods}

Plant Materials. The leaves of six citrus varieties (Table 1) were used in this experiment, all provided by the Citrus Research Institute of Huazhong Agricultural University.

Table 1 used in six citrus germplasm materials for testing

\begin{tabular}{lll}
\hline Germplasm & Latin Name & Classification $^{[6]}$ \\
\hline Nanfeng tangerine & C. reticulata Blanco cv. Kinokuni & Sinocitrus \\
Eureka lemon & C. limon (L.) Burm. F. & Citrophorum \\
Gaoban pummelo & C. maxima (Burm.) Merr. & Cephalocitrus \\
Kiyomi & C. unshiu Marcov. $\times$ C. sinensis Osbeck & Miscellaneous Citrus \\
Ichang papeda & C. ichangensis Swingle & Papedocitrus \\
Ponkan & C. reticulata Blanco cv. Ponkan & Sinocitrus \\
\hline
\end{tabular}


SSR-PCR system and electrophoresis. The DNA of 6 citrus leaf samples was extracted and detected using the kit Plant Genomic DNA Kit (TIANGEN, Beijing) and stored at $-20{ }^{\circ} \mathrm{C}$ for later use. A total of $20 \mu \mathrm{L}$ of PCR reaction system was prepared. The PCR amplification product was electrophoresed on $6 \%$ denaturing polyacrylamide gel and stained with silver nitrate.

Statistical Methods. The number of alleles of electrophoresis bands was counted, and the polymorphic ratio of each primer was calculated; SSR electrophoresis showed a band of 1, no stripe was 0 , stripe deletion was 2 , and 1 and 0 matrices were obtained. The similarity coefficient was calculated by NTSYS-pc2.10e software, and the similarity coefficient matrix ${ }^{[7]}$ was obtained. The SAHN program and UPGMA were used to cluster analysis ${ }^{[8]}$ for the sample of citrus.

\section{Results}

Product characteristics of SSR-PCR amplification. In the 'Nanfeng tangerine', there are 23 pairs of primers to amplify the band, 21 pairs of primers in 'Eureka lemon' to amplify the band, and 22 pairs of 'Gaoban Pomelo' have amplified bands, There were 23 pairs of amplified bands in the ' Kiyomi', and 20 pairs of 'Ichang papeda' amplified the bands, and 25 pairs of 'Ponkan' amplified the bands. 26 pairs of primers in 38 pairs successfully amplified clear bands (Table 2), and 12 pairs of primers did not amplify bands. The total number of amplified bands of 26 pairs of SSR primers amplified was 81 , and average of 3.12 bands were amplified per primer. The total number of bands per primer ranges from 1 to 6 , that is, the number of alleles is 1-6.A total of 72 polymorphic bands were amplified, and an average of 2.77 polymorphic bands were amplified per primer. The polymorphic bands of the primers amplified by the test varied greatly, and the polymorphism ratio ranged from 50.00-100.00\%. The SSR primers screened from 20 pairs of citrus were clear and polymorphic (polymorphic ratio was $66.67 \% \sim 100.00 \%$ ). The target fragments of 13 pairs of primers are 150-200 bp in length; the selected 20 pairs of SSR primer target fragments are concentrated in the range of 150-250 bp.

Table 226 pairs of SSR primer sequences

\begin{tabular}{lccccc}
\hline $\begin{array}{l}\text { Primer } \\
\text { ID. }\end{array}$ & $\begin{array}{c}\text { Number of } \\
\text { PCR band }\end{array}$ & $\begin{array}{c}\text { Number of polymorphic } \\
\text { PCR band }\end{array}$ & $\begin{array}{c}\text { Polymorphi } \\
\text { sm ratio (\%) }\end{array}$ & $\begin{array}{c}\text { Expected } \\
\text { amplicon (bp) }\end{array}$ & $\begin{array}{c}\text { Reference } \\
\text { source }\end{array}$ \\
\hline CS 010 & 3 & 3 & 100.00 & $150-200$ & {$[9]$} \\
CS 065 & 4 & 4 & 100.00 & $150-200$ & {$[9]$} \\
CS 062 & 3 & 3 & 100.00 & $200-250$ & {$[9]$} \\
CS 009 & 3 & 2 & 66.67 & $150-200$ & {$[9]$} \\
CS 100 & 3 & 3 & 100.00 & $200-250$ & {$[9]$} \\
CS 051 & 3 & 3 & 100.00 & $150-200$ & {$[9]$} \\
CS 021 & 3 & 3 & 100.00 & $200-250$ & {$[9]$} \\
CS 024 & 6 & 6 & 100.00 & $150-250$ & {$[9]$} \\
CS 017 & 6 & 6 & 100.00 & $150-250$ & {$[9]$} \\
CS 052 & 2 & 2 & 100.00 & $150-200$ & {$[9]$} \\
CS 064 & 3 & 3 & 100.00 & $150-200$ & {$[9]$} \\
CS 068 & 3 & 3 & 100.00 & $200-250$ & {$[9]$} \\
CS 014 & 2 & 1 & 50.00 & - & {$[9]$} \\
AAT 12 & 3 & 2 & 66.67 & $150-200$ & {$[10]$} \\
TAA 45 & 3 & 3 & 100.00 & $100-150$ & {$[10]$} \\
TAA 15 & 4 & 4 & 100.00 & $150-200$ & {$[10]$} \\
CMS 30 & 5 & 5 & 100.00 & $150-200$ & {$[10]$} \\
AG 16 & 5 & 5 & 100.00 & $150-200$ & {$[10]$} \\
CMS 7 & 2 & 2 & 100.00 & $150-200$ & {$[10]$} \\
CMS 45 & 3 & 3 & 100.00 & $150-200$ & {$[10]$} \\
TAA 1 & 3 & 3 & 100.00 & $150-200$ & {$[10]$} \\
\hline
\end{tabular}




\begin{tabular}{lllcll}
\hline TAA 27 & 2 & 1 & 50.00 & - & {$[10]$} \\
CMS 8 & 1 & 0 & 0.00 & - & {$[10]$} \\
CMS 14 & 2 & 1 & 50.00 & - & {$[10]$} \\
CMS 20 & 2 & 1 & 50.00 & - & {$[10]$} \\
CT 21 & 2 & 0 & 0.00 & - & {$[10]$} \\
\hline
\end{tabular}

Cluster analysis of citrus cultivars for trial.The combination of 'Eureka lemon' and 'Ichang papeda' has a genetic similarity of 0.74 , which indicates that there is a close relationship between the two. This type of 'Ponkan Citrus' is a class, the genetic similarity coefficient is 0.69 , with 'Nanfeng tangerine' aggregation, the genetic similarity coefficient is 0.59 . 'Gaoban pomelo' and 'Kiyomi' gather for another independent group, the genetic similarity coefficient of 0.71 , indicating that the blood relationship between the two is close.

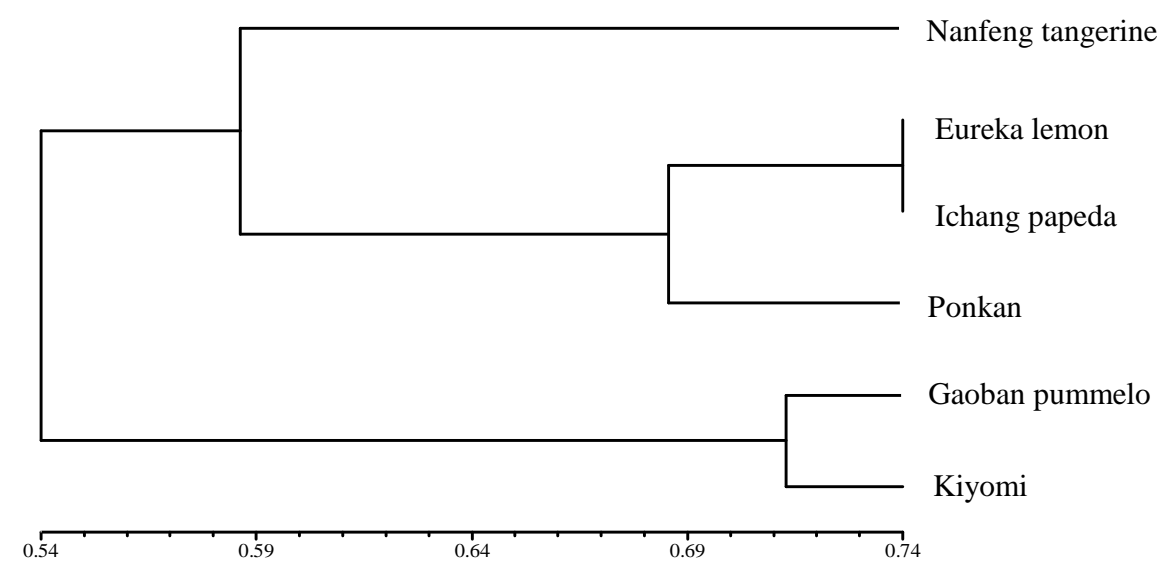

Fig.2 Dendrogram of six citrus materials clustering analysis

Discussions. 'Eureka Lemon' and 'Ichang papeda' are grouped together, supporting Xiao et al. ${ }^{[11]}$ through cytology, Zhu et al. ${ }^{[12]}$ through chemical taxonomy and Federici et al. ${ }^{[13]}$,It is one of the parents of the origin of lemon hybrids. The pupa and pummelo proposed by DNA molecules may be derived from the big wing orange. Barrett and Rhodes ${ }^{[14]}$ concluded that sweet orange is produced by the hybridization of pummelo with different subspecies of polytypic species; Liang et al. ${ }^{[15]}$ supported the hybrid origin of sweet orange by cytological studies and believed that pummelo is parent, Malix et al. ${ }^{[16]}$ supported the broad-skin citrus as one of parents through protein electrophoresis analysis. The 'Kiyomi' is a descendant of Wenzhou mandarin as the female parent and sweet orange as the male parent $^{[17]}$, which is consistent with the previous research conclusions, may be derived from the big wing orange, speculative lemon and 'Ichang papeda' have a closer blood relationship; 'Gaoban pummelo' and 'Kiyomi' are grouped together, seeing the father is sweet orange, $\mathrm{Xu}$ et al. ${ }^{[18]}$ verified the pummelo as a sweet orange parent. This experiment speculated that 'Gaoban pummelo ' has close kinship with 'Kiyomi' and provided support for the conclusions of predecessors; The genetic similarity coefficient of 'Ponkan' is 0.68 with 'Eureka Lemon' and 'Ichang papeda', although ponkan is the offspring of orange, and the AFLP distance tree of Xie et al. ${ }^{[19]}$ supports the viewpoint of the origin of broad-skin citrus single line, so it gathering into another category; 'Nanfeng tangerine' has a relatively long genetic distance from other citrus varieties, and it is also clustered into a large group.

\section{Conclusions.}

This study screened 20 pairs of primers with high polymorphism, clear and easy-to-recognize bands, and good repeatability. According to the cluster analysis, 'Eureka Lemon' and 'Ichang papeda' are grouped together, 'Gaoban pummelo , ' and 'Kiyomi' are grouped together, 'Ponkan' and 'Nanfeng Tangerine' and others The genetic distance of citrus varieties is relatively far, and they are separately grouped into one group. 


\section{References}

[1] Z.K. Liao: Evaluation of citrus germplasm resources in Hunan and main diseases molecular detection. Hunan Agricultural University (2007)

[2] L.Zhang, X.P. Ding and L.L.Yan: Optimization of SSR-PCR system and primers screening of eight species of Stylosanthes. Pratacultural Science Vol. 31(2) (2014), p. 232-242

[3] T.G. Lei, Y.R. He and X. Wu: Construction of DNA fingerprinting database of citrus cultivars (lines). Scientia Agricultura Sinica Vol. 42(8) (2009), p. 2852-2861

[4] H.B. Ma, X.M. Xu and X.Y. Wei: DNA fingerprints and genetic diversity analysis based on SSR markers for rice cultivars in Fujian. Fujian Journal of Agricultural Sciences Vol. 25(1) (2010), p. 33-38

[5] L.U. Dan, N. Niu and Y.Y. Li: Application of SSR Marker in Plant Genome Research. Journal of Shenyang Normal University Vol. 28(1) (2010), p. 83-85

[6] K.L. Zhou and M.M. Ye: Chinese fruit tree (Citrus reel). China Forestry Publishing House (2010)

[7] F.J. Rohlf: nvimerieal taxonomy and multivariate analysis system,version 2.1. Exeter Publications (2000)

[8] P.H. Sneath: Classification of microorganisms. John Willey and Sons Ltd, Sydney (1978)

[9] H. Li, X.M. Yang and L.S. Zhu: Parentage analysis of natural citrus hybrid'Zhelong Zhoupigan'based on nuclear and chloroplast SSR markers. Scientia Horticulturae Vol. 186 (2015), p.24-30

[10] T. Liu: Genetic Diversity Analysis of Guangxi Citrus Germplasms Using SSR and SRAP. Huazhong Agricultural University (2014)

[11] Z.A. Xiao: REVIEW ON "CITRUS THREE-GENERA LEFT-RIGHT CLASSIFICATION SYSTEM". 1 Vol. 3 (1984), p. 44-50

[12] L.W. Zhu: Numerical chemotaxonomical study on Citrus plants cultivated in China. Acta Phytotaxonomica Sinica Vol. 26 (1988), p. 353-361

[13] C.T. Federici: Phylogenetic relationships within the genus Citrus (Rutaceae) and related genera as revealed by RFLP and RAPD analysis. Theoretical and Applied Genetics Vol. 96 (1998), p. $812-822$

[14] H.C. Barrett and A.M. Rhodes: A numerical taxonomic study of affinity relationships in cultivated Citrus and its close relatives. Systematic Botany Vol. 1 (1976), p. 105-136

[15] G.L. Liang: Studies on the cytotaxonomy of citrus I. Karyotype and evolution of 30 taxa of the genera citrus. Journal of Wuhan Botanical Research Vol. 8 (1990), p. 1-7

[16] M.N. Malix, R.W. Scora and R.K. Soost: Studies on the origin of the lemon. Hilgardia Vol. 42 (1974), p. 361-382

[17] F.L.Weng: Kiyomi and its utilization in citrus breeding. South china fruit Vol. 33(5) (2004), p. 8-9

[18] Q. Xu, L.L. Chen and X. Ruan: The draft genome of sweet orange (Citrus sinensis). Nature Genetics Vol. 45(1) (2013), p. 59-66

[19] R.J. Xie, Z.Q. Zhou and L. Deng: Taxonomic and phylogenetic relationships among the genera of the True Citrus Fruit Trees Group (Aurantioideae, Rutaceae) based on AFLP markers. Journal of Systematics \& Evolution Vol. 46(5) (2008), p. 682-691 